\title{
AN OBJECT PROPERTIES FILTER FOR MULTI-MODALITY ONTOLOGY SEMANTIC IMAGE RETRIEVAL
}

\author{
Mohd Suffian Sulaiman, Sharifalillah Nordin \& Nursuriati Jamil \\ Centre for Computer Science Studies, \\ Universiti Teknologi MARA, Malaysia
}

suffian@tmsk.uitm.edu.my; sharifa@tmsk.uitm.edu.my; liza@tmsk.uitm.edu.my

\begin{abstract}
Ontology is a semantic technology that provides the possible approach to bridge the issue on semantic gap in image retrieval between low-level visual features and high-level human semantic. The semantic gap occurs when there is a discrepancy between the information that is extracted from visual data and the text description. In other words, there is a difference between the computational representation in machine and human natural language. In this paper, an ontology has been utilized to reduce the semantic gap by developing a multi-modality ontology image retrieval with the enhancement of a retrieval mechanism by using the object properties filter. To achieve this, a multi-modality ontology semantic image framework was proposed, comprising of four main components which were resource identification, information extraction, knowledge-based construction and retrieval mechanism. A new approach, namely object properties filter is proposed by customizing the semantic image retrieval algorithm and the graphical user interface to facilitate the user to engage with the machine i.e. computers, in order to enhance the retrieval performance. The experiment results showed that the proposed approach delivered better results compared to the approach that did not use the object properties filter based on probability precision measurement.
\end{abstract}

Keywords: Multi-modality ontology, semantic image retrieval, object properties, semantic gap, object properties filter. 


\section{INTRODUCTION}

With the current advancement of technology, the image retrieval (IMR) have become important in research over the last four decades as there was a need to control and manage the collection of large images effectively (Rui, Huang, \& Chang, 1999). The initial stage of the IMR method was called the text-based image retrieval (TBIR), which used text associated with a certain image to determine what the image contained (Riad, Elminir, \& Abd-Elghany, 2012). Due to several disadvantages of the TBIR approach, such as no standard image filling and consuming a lot of human effort, the content-based image retrieval (CBIR) was proposed. In CBIR, the images were retrieved through the indexing of low-level features such as colour, texture and shape. There were many algorithms that have been developed to describe the low-level features (Zhang, Islam, \& Lu, 2012). However, these algorithms failed to model the image semantics as how humans interpret the images (Zhang, 2007). Therefore, the semantic-based image retrieval (SBIR), had been proposed as a possible solution to bridge the semantic gap (Smeulders, Worring, Santini, Gupta, \& Jain, 2000) between low-level features and highlevel human semantic. A review was done by Liu, Zhang, Lu and Ma (2007) who discovered that ontology is one of the techniques available to reduce the semantic gap. Therefore, this study developed the multi-modality ontology by assimilating the textual information and visual features to high-level concepts so that humans and machines were complementary. In this study, a herbal medicinal plant was chosen as the case study since there is a significant demand from people around the world. The World Health Organization (WHO) (WHO, 2008) stated that between $70 \%$ to $80 \%$ of developed countries used alternative medicines for health purposes. This high percentage portrays the crucial need of semantic descriptions for the herbal medicinal plant images to cater for various users' information needs. Moreover, this study explored the significance and the impacts of object properties filter which can be exploited in the retrieval mechanism in order to achieve the main goals in IMR, to increase the relevance and accuracy of digital images retrieval.

\section{RELATED WORKS}

Ontology can be defined as a formal depiction of a set of entities within a domain and the relationship among those entities. A formal ontology comprised of a controlled vocabulary articulated in a representation language. This language had syntax in using vocabulary terms to label something meaningful within the interests of a specified domain (Gruber, 1993). In the context of IMR research, 
ontology was used to define the high-level concepts using well-structured approaches and relationships that were human readable and meaningful (Liu et al., 2007). For example, in some IMR systems, image descriptors were used to form a simple vocabulary such as light green, medium green and dark green (Mezaris, Kompatsiaris, \& Strintzis, 2003). It provides a qualitative definition of high-level query concepts which were understood by humans. Therefore, ontology could assist machines in analyzing semantic visual information from various perspectives and provide unlimited descriptive power of semantics.

\section{Ontology and Image Retrieval System}

Some earlier CBIR proposed the concept of integrating high-level textual information and low-level features without considering the requirement of ontology which had the capability of providing an explicit domain-oriented semantics in terms of defining concepts and their relationships. Therefore, the multi-modality ontology approach was proposed to enhance the previous studies in order to achieve the main goal in IMR. This concept was then exploited by several researchers in their respective IMR work. Multi-modality ontology was the integration of high-level textual information and low-level image features metadata to represent the image contents for image retrieval (Wang, Liu, \& Chia, 2008). A number of studies (Khalid., Azman, \& Noah, 2012; Kesorn, 2010; Singh, Goudar, Rathore, Srivastav, \& Rao, 2013; Magesh \& Thangaraj, 2011; Wang et al., 2008) have proven that the multi-modality ontology could improve the retrieval performance to obtain more accurate results. Previously, researchers were prone to adapt the single-modality approaches, either TBIR or CBIR. However, due to the limitation of both approaches, the need to combine both approaches to form a multi-modality or hybrid approach had become critical. Wang et al. (2008) performed an experiment by comparing the traditional keyword-based, single text ontology and multi-modality ontology. By using 4000 canine domain images as a sample, the authors found that the retrieval performance had improved by about 5 to 30 percent by combining the high-level textual information with low-level image features and introducing the domain ontology into the multi-modality ontology as an important cue to solve the problems of semantic interpretation in image retrieval. Therefore, the multi-modality ontology could provide better retrieval results compared to the single ontology. However, without a proper method to control the targeted images, the retrieval result would provide many irrelevant images, thus, decreasing retrieval accuracy. Khalid et al. (2012) proposed an improvement by integrating the multi-modality ontology with DBpedia. The proposed method improved the performance of 
image retrieval by interlinking it with the Linked Data technology. The web developers could make use of the rich source of information and the domain ontology to enrich their vocabulary control. However, when such ontology is integrated with too much enriched vocabulary, especially the DBPedia which contains 2.6 million entities and 4.7 billion pieces of information, the performance of the precision rate would be low when many unrelated images results returned from the queries. Khodaskar \& Ladhake, (2015) embarked on the image retrieval system using an alignment of ontologies in order to reduce the semantic gap and provide highly accurate, efficient and effective image retrieval results. They proposed multiple ontologies which merged feature ontology, semantic feature ontology, user ontology and metadata ontology to overcome the traditional image retrieval system that used the single ontology which retrieved imprecise images. The proposed system used image query as input via ontology language interface which was implemented to reduce the semantic gap, and then features were extracted and mapped by the proposed ontology. However, there was no approach to filter the retrieval result even though the problem of a single modality might be resolved by using multiple ontologies. The non-filter image results would affect the accuracy of retrieval especially when the results of queried images were enormous. Singh et al. (2013) utilized the multi-modality ontology concept for the development of IMR for their respective image on medicinal dataset. However, the authors did not look in detail the retrieval mechanism component for means of filtering for more accurate results. When the users were confronted with the big scale of metadata, the possibility of retrieval result being ernomous, cannot be denied. As a result, it would decrease the performance of the retrieval images. Despite the fact that applying the multi-modality ontology in IMR showed better results by comparing them with the keyword-based and the single ontology, there was still a gap in order to achieve the optimum result especially on the retrieval mechanism. Great attention was given to enrich the vocabulary of ontology to resolve the lack of metadata depth with the hope that users would retrieve the image successfully. The multi-modality ontology approach resolved the issue of incompleteness in ontology metadata which caused many that queries would return the null image results and would affect the precision rate to be low. However, using the multi-modality approach, would cause too much vocabulary in ontology and return many irrelevant results which were also responsible for a low image precision rate. Therefore, there was a need to have a mechanism that could control or filter the vocabulary which would improve the precision rate. This paper discusses the proposed approach by incorporating the multi-modality ontology and herbal medicinal plant dataset with the object properties filter approach. 


\section{Relevant Feedback in Image Retrieval}

The early works of relevance feedback approach had been identified during the 1960s which traditionally used the text-based information retrieval system (Rocchio, 1966). In mid-1990s, the relevance feedback had attracted the CBIR community in an effort to reduce the semantic gap between the representation of the queries in low-level features and the way a user thinks of high-level concepts (Kurita \& Kato, 1993). Numerous solutions have been proposed and have remained as active research topics to this day. The significance of relevant feedback in image retrieval was due to the existence of more ambiguities when interpreting the images than the words, and judging the images took too much time. Thus, there was a necessity for user interaction involvement which expedited the feedback process because an image often reveals its content almost instantly to a human observer (Zhou \& Huang, 2003). A typical scenario of relevance feedback in a content-based image retrieval (Zhou \& Huang, 2003) could be carried out as follows:

1. The user performs the query through query-by-keyword, sketch, etc. and an initial retrieval result is provided by the system.

2. Based on the initial retrieval result, the user judges whether it is relevant or irrelevant to their query.

3. Machine learning algorithm is utilized to learn the user feedback. Then, return to number 2 .

The second and third processes were repeated until the user was satisfied with the final results. Six common approaches were used in the relevance feedback: Query Re-weighting (QR), Query Point Movement (QPM), Query Expansion (QE), Log-based Relevance Feedback, Navigation Pattern Relevance Feedback (NPRF) and Particle Swarm Optimization Relevance Feedback (Sivakamasundari \& Seenivasagam, 2012). The following sections will discuss each of the approaches used in image retrieval.

\section{Query Reweighting}

The earlier work of relevance feedback in CBIR was query reweighting. The main idea of QR was that if the $i_{\text {th }}$ feature $f_{\mathrm{i}}$ existed in most of the positive examples, the system was assigned to the higher degree to $f_{\mathrm{i}}$ (Rui, Huang, $\&$ Mehrotra, 1997). In other words, the adjustment of the low-level feature weight was needed to accommodate the user's need. The disadvantage of this approach is that the image retrieval is very limited due to the diverse visual feature and some targeted images could be missing. 


\section{Query Point Movement}

This concept was based on Rocchio's formula (Rocchio, 1965) as an examplebased query refinement. One of the QPM approaches was PicHunter (Cox, Miller, Minka, Papathomas, \& Yianilos, 2000) which made use of the Bayesian prediction to infer from targeted images. Even though QPM tries to improve the estimate of the ideal query point by moving towards positive examples, using a single measuring function would not be able to cover all the targeted groups with various visual contents. Thus, it is hard for QPM to reach global optimal results.

\section{Query Expansion}

Since QR and QPM were not able to improve the performance of relevance feedback, the researchers moved on to QEX as a potential solution. QEX is a process of reformulating a seed query in order to improve the retrieval performance in information retrieval. Two methods could be used in QEX. Firstly, using global methods by expanding or reformulating query independent of the query and the results returned. Secondly, local methods adjust the query relative to the image that initially appears to match the query. One example of QEX is Qcluster, developed by Kim \& Chung (2003). The authors proposed handling the disjunctive queries by employing adaptive classification and cluster merging methods. The proposed Qcluster showed that the effectiveness of QEX was better than QPM and QR in terms of retrieval quality.

\section{Log-based Relevance Feedback}

The log-based relevance feedback incorporated the log data of users' relevance feedback with regular relevance feedback for image retrieval (Hoi, Lyu, \& Jin, 2006). In the proposed architecture, if the feedback log data was unavailable, the log-based relevance feedback algorithm would behave exactly like a regular relevance feedback algorithm. Otherwise, if the feedback log data was available, the algorithm would learn the correlation using both feedback $\log$ data and the online feedback from the users. Therefore, the log-based relevance feedback approach had the ability to complete the retrieval goal in only a few iterations with the support from the log data of users' feedback.

\section{Navigation Pattern Relevance Feedback}

The NPRF is proposed to reduce the iterations of feedback in the existing relevance feedback approach. Therefore, NPRF can be used in real applications practically and efficiently ( $\mathrm{Su}$, Huang, \& Yu, 2011). NPRF utilized the 
discovered navigation patterns and three types of query refinement strategies which are QR, QPM and QEX in order to achieve high performance of image retrieval with a small number of feedbacks.

\section{Particle Swarm Optimization Relevance Feedback}

The particle swarm optimization relevance feedback was proposed to address three main issues. Firstly, the user interaction was time consuming and tiring due to a much number of iterations of convergence. Secondly, only a small number of new or possibly none images were retrieved during the first relevance feedback steps with no positive examples available for successive retrieval, and thirdly, the risk of stagnation. In other words, the inability to further discover the image space was due to the search process converging to below optimal local solution (Broilo \& Natale, 2010).

The most current works in relevance feedback approach proposed a new form of feedback by using relative attributes (Kovashka, Parikh, \& Grauman, 2015). This approach allowed the user to precisely indicate how the result compared with their mental mode. In other words, the users were able to describe which properties of exemplar images should be adjusted in order to more closely match their mental mode of the image required. The author claimed that their system-guided approach could rapidly pinpoint the visual target using a series of well-chosen comparative queries.

The first three approaches QR, QPM and QEX were the pioneered concepts of relevance feedback in image retrieval. The QEX was better than QPM and QR (Sivakamasundari \& Seenivasagam, 2012). However, the remaining approaches were incomparable even though they were placed under the similar idea of "relevance feedback". It was because the different methods depended on particular expectations or problem backgrounds (Zhou \& Huang, 2003). In this research, the typical scenario of the relevance feedback concept was exploited and customized by proposing a new approach called object properties filter. In this approach, the user could perform the query using query-by-keyword and the system would provide the initial results similar to a typical scenario of the relevance feedback. Then, based on the initial results, the user could judge the relevancy of the image by selecting an appropriate relationship or object properties listed with initial image results. However, no machine learning was utilized as a typical scenario of the relevance feedback concept. It was due to the fact that the proposed approach was prone to screening the initial results based on particular object properties related to user query and not measuring the similarity of the selected images. Eventually, the optimized final result would be displayed to the user. 


\section{Object Properties}

Object properties represent the relationships between two individuals in ontology. In common practice, the name of an object property starts with a lower case letter, has no spaces, has remaining words capitalized and the name is unique. It is also recommended that the properties are prefixed with the word 'has' e.g. hasShapeFlower, hasFruitType, and hasRootHabit. Usually, object properties are used in establishing relationships in ontology (Noy \& McGuinness, 2000). However, this study exploited the object properties to filter the initial retrieval results to be more accurate in terms of precision. For example, if our metadata had a similar name in different classes, the results would reduce the performance of precision. Therefore, the object properties could be utilized to filter more accurate retrieval results and improve the performance of precision.

\section{METHODOLOGY}

\section{The Framework}

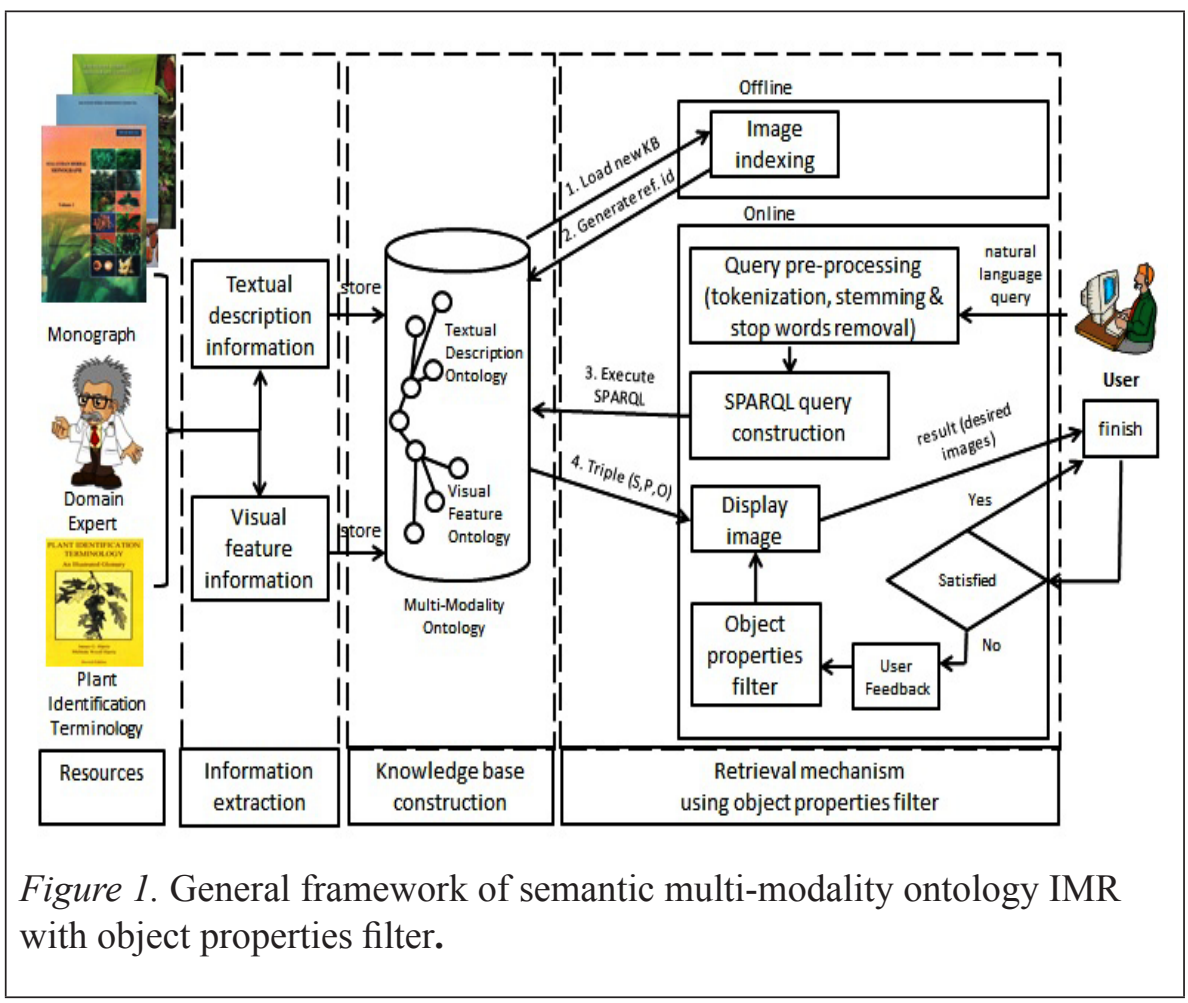


Figure 1 depicts the general framework of the multi-modality ontology IMR system which consists of four main components which are resources, information extraction, knowledge base construction and retrieval mechanism using object properties filter.

\section{Resources Component}

The resources component represents the identification of the domain, scope, corpora and metadata. The domain experts in the herbal medicinal plant were consulted in order to clarify the contents of the corpora (Commitee, 2009, 2012; Ismail, Ismail, \& Lassa, 1999). The plant identification terminologies were referred (Harris \& Harris, 2004) to describe the classification of the plant concept during the process of analyzing the potential class.

\section{Information Extraction Component}

Two types of information extraction were performed which analyzed the textual description information and interpreted the semantic visual feature information. The textual description information described the high-level concepts of the images. The textual information could be the facts from the corpora that described the particular herbal medicinal plant images. The extraction work began with collecting the information from the monographs with the assistance of the domain experts and by referring to the plant identification terminology. Other than that, formal and informal interviews, brainstorming, and formal and informal analysis were performed with the domain experts. All possible terms needed were jotted down. Then, the table template was constructed to enumerate the important terms in ontology, namely as the plant term worksheet. This worksheet was used in organizing the concept term. It was an output of the knowledge extraction for textual description information. To extract the semantic visual feature information, the method proposed by Wang et al. (2008) was adapted. Firstly, the set of terms adapted from the corpora that were relevant to the image content was defined. Then, the visual feature information that described the low-level features information based on the particular herbal medicinal plant image was extracted. In this study, the compact composite low-level features descriptors were used to extract images such as Colour and Edge Directivity Descriptor (CEDD), Brightness and Texture Directionality Histogram (BTDH) and Fuzzy Colour and Texture Histogram (FCTH) because these outperform the MPEG7 descriptors (Chatzichristofis, Zagoris, Boutalis, \& Papamarkos, 2010). After that, all the images were classified according to the XML index data from the extracted images by using supervised machine learning. The Bayesian network 
classifier was used since it could provide effective knowledge representation and as an inference engine in artificial intelligent (Serrano, Savakis, \& Luo, 2004). Finally, each image had a set of tags to describe its content, which were matched with the concepts defined in the visual feature ontology.

\section{Knowledge Base Construction Component}

Once all the textual description and visual feature information were extracted, the construction of the ontology could be implemented. In order to construct the ontology, three important factors had to be defined: the classes, properties and instances. In this study, the top-down approach from Uschold and Gruninger (1996) was adapted. This approach started by identifying the most general concept in the domain and then continued with more specialized concepts. Several classes were defined, including Plant, Character, Odour, Physical, Taste, GeneralHabit and LeafPart. The semantic relationships were generated to connect the variety of concepts such as hasColourFlower, hasFruitHabit, hasFlowerPart and hasLeafBases. Finally, the relationship between TextualConcept and VisualConcept to be the multi-modality ontology, was manually created as illustrated in Figure 2.

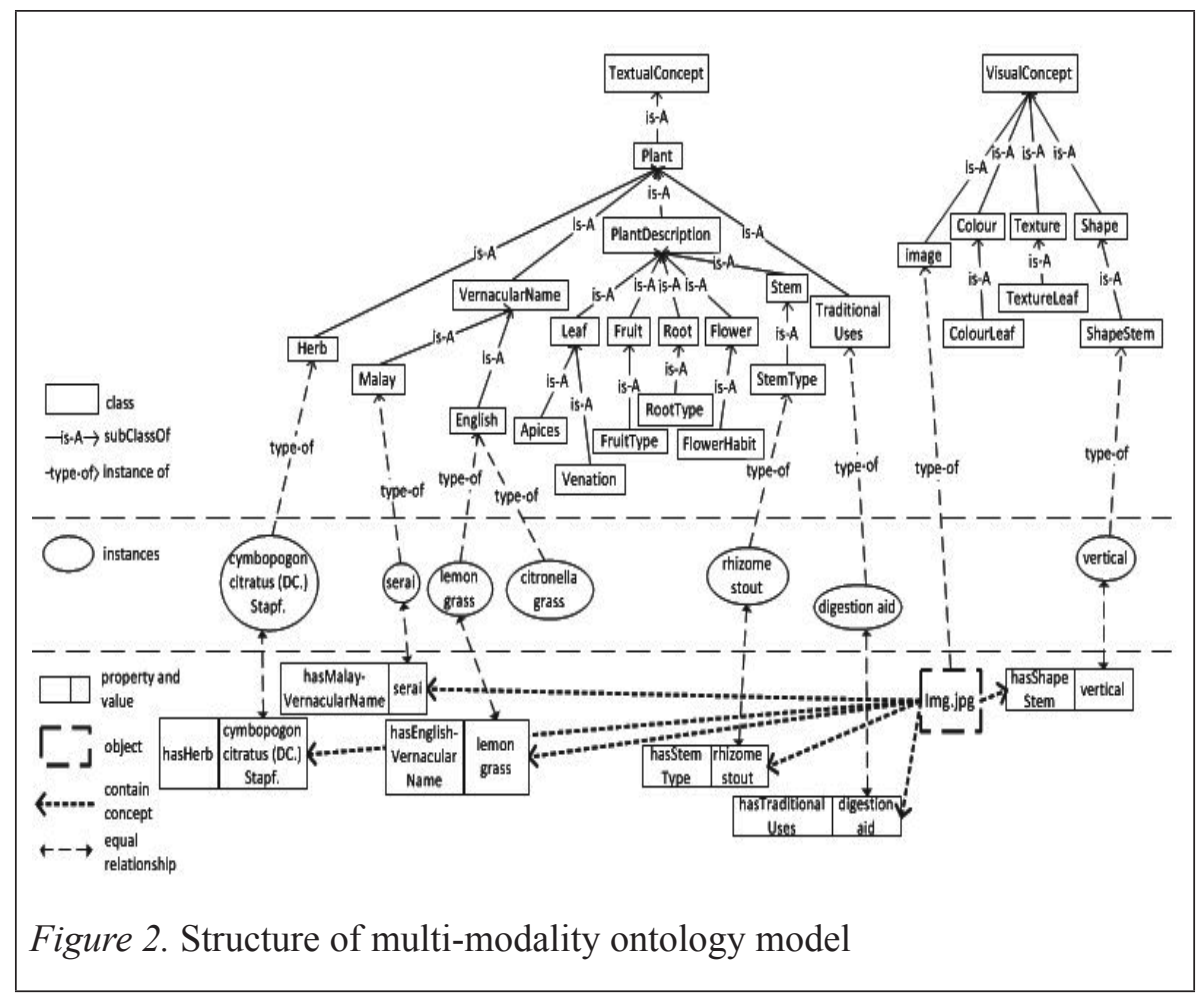




\section{Retrieval Mechanism using Object Properties Filter Component}

In the retrieval mechanism component, the natural language interface approach was utilized where the user could enter the natural language query into the interface. The natural language interface returns the desired images as required to the user if matched. Before the images could be retrieved from the multi-modality ontology, the images need to be indexed first. In the offline side, the multi-modality ontology needed to be loaded first, then the images were indexed by generating five digit unique numbers as their reference id according to their image paths. The Jena application programming interface (API) embedded with the ARQ query engine was employed to construct the search engine. In this study, the Liferay platform was used to receive the natural language input query, to index the images and display a set of matching images as a result to the user. The algorithm of a semantic multi-modality ontology IMR system given in Figure 3 is adapted from Kesorn (2010) with modification shown in steps 3.1 to 4 .

(1) Give the input query in natural language.

(2) Perform the query pre-processing, e.g. tokenization, stop word removal and stemming (Saian \& Ku-Mahamud, 2004) to get a set of relevant query keywords.

(3) Based on the relevant query keywords, construct the Simple Protocol and RDF Query Language (SPARQL).

(3.1) Identify the namespace of the multi-modality ontology.

(3.2) Identify the selection of the variables with the list of object properties.

(3.3) Identify the instance based on related object (metadata).

(3.4) Identify the image instance in the image class.

(3.5) Identify the image id based on the image instance.

(3.6) Identify the object properties according to image instance and instance.

(3.7) Identify the label of object properties to display on the GUI.

(3.8) Filter the set of relevant query keywords.

(3.9) Execute SPARQL based on identified instance, object, image instance, image id and object properties.

(4) If the query pattern did not match the multi-modality ontology then

Relevant images result $=$ null else,

Display the relevant images result based on the highest similarity score with dynamic object properties.

Figure 3. Semantic multi-modality ontology IMR system algorithm. 
(5) If the user is not satisfied with the initial retrieval result, then, Receive feedback from the user by selecting the dynamic object properties. Display the updated retrieval result.

else,

Terminate the retrieval process.

Figure 4. Algorithm for object properties filter.

In this algorithm, the list of object properties according to the user query was identified (see Figure 3 (3.2)). Different user query input would produce a different list of object properties. The list of object properties was displayed to the user on the graphical user interface (GUI) together with the list of initial images result. In order to improve the precision of retrieval, a new approach was added to the existing algorithm which involved humans as a part of the retrieval process as shown in Figure 4. This concept was quite similar to the relevance feedback (Sivakamasundari \& Seenivasagam, 2012) approach. However, in the relevance feedback, the user marked the queried images as a searched image that they wanted, but in this approach, the user would mark the object properties that were dynamically displayed with the queried images in the interface. The object property was marked based on the relevance of the relationship and the judgement of the user to the query. For example in Query 2: Find herbal medicinal plant that has aromatic smell? The lists of object properties were hasOdourCharacter, hasFruitHabit, hasLeafHabit and hasSynonym. In this case, based on the relevant user judgement, hasOdourCharacter was the most relevant object property compared to the others. Then, the marked object properties were fed back into the system as a new redefined query for the following cycle of the retrieval process.

\section{RESULTS AND DISCUSSION}

To evaluate the retrieval performance of the proposed semantic multi-modality ontology IMR, 60 species of herbal medicinal plants were selected with 1114 instances, 3384 triples, 46 object properties, 61 classes and 180 images comprising the whole plant, leaf, flower, slender, stem, fruit, seed, root, bud, aerial and underground part images. The retrieval precision measurement was used where the numbers of relevant documents retrieved were divided by the total number of documents retrieved in that search. Let ' $A$ ' denote all relevant 
images (as specified in a user query) in the image collection and ' $B$ ' denote the retrieved images which the system returns for the user query. Precision $(\mathrm{P})$ is defined as the portion of relevant images in the retrieved image:

$$
\operatorname{Precision}(P)=\frac{|A \cap B|}{|B|}
$$

The image retrieval using the object properties filter and retrieval without using the object properties filter was compared to benchmark the performance. Ten natural language queries as competency questions (Noy \& McGuinness, 2000) were selected. Table 1 shows the list of queries.

Table 1

\section{List of Evaluation Queries}

\begin{tabular}{ll}
\hline Query no. & Natural language query \\
\hline Q1 & What herbal medicinal plant has entire leaf margins? \\
Q2 & Find herbal medicinal plant that has aromatic smell? \\
Q3 & What herbal medicinal plant can treat cough diseases? \\
Q4 & Find herbal medicinal plant that has elliptic shaped leaf? \\
Q5 & What herbal medicinal plant has ovoid shaped fruit? \\
Q6 & Find herbal medicinal plant that has acuminate leaf apices? \\
Q7 & What herbal medicinal plant has hard tubers? \\
Q8 & Find herbal medicinal plant that has rhizome type? \\
Q9 & What herbal medicinal plant has bitter taste? \\
Q10 & Find herbal medicinal plant that has tree features? \\
\hline
\end{tabular}

Table 2

Evaluation Results

\begin{tabular}{|c|c|c|c|c|c|c|c|c|}
\hline \multirow{2}{*}{$\begin{array}{l}\text { Queries } \\
\text { No. }\end{array}$} & \multicolumn{4}{|l|}{ MMO-IMR } & \multicolumn{4}{|c|}{ MMO-IMR-OPF } \\
\hline & OP & $\mathrm{C}$ & $\mathrm{R}$ & $P$ & OPF & $\mathrm{C}$ & $\mathrm{R}$ & $\mathrm{P}$ \\
\hline Q1 & $\begin{array}{l}\text { hasLeafPart, hasLeafMargins, } \\
\text { hasLeafHabit, hasLeafDivision }\end{array}$ & 14 & 21 & 0.6667 & hasLeafMargins & 14 & 14 & 1.0000 \\
\hline Q2 & $\begin{array}{c}\text { hasOdourCharacter, } \\
\text { hasFruitHabit, hasLeafHabit, } \\
\text { hasSynonym }\end{array}$ & 23 & 36 & 0.6389 & hasOdourCharacter & 23 & 33 & 0.6970 \\
\hline Q3 & hasTraditionalUses & 6 & 9 & 0.6667 & hasTraditionalUses & 6 & 9 & 0.6667 \\
\hline
\end{tabular}




\begin{tabular}{|c|c|c|c|c|c|c|c|c|}
\hline \multirow{2}{*}{$\begin{array}{l}\text { Queries } \\
\text { No. }\end{array}$} & \multicolumn{4}{|l|}{ MMO-IMR } & \multicolumn{4}{|c|}{ MMO-IMR-OPF } \\
\hline & OP & $\mathrm{C}$ & $\mathrm{R}$ & $\mathrm{P}$ & OPF & $\mathrm{C}$ & $\mathrm{R}$ & $\mathrm{P}$ \\
\hline Q4 & $\begin{array}{l}\text { hasShapeLeaf, hasLeafHabit, } \\
\text { hasLeafPart, hasShapeFlower }\end{array}$ & 22 & 31 & 0.7097 & hasShapeLeaf & 22 & 22 & 1.0000 \\
\hline Q5 & hasDefinition, hasShapeFruit & 4 & 12 & 0.3333 & hasShapeFruit & 4 & 4 & 1.0000 \\
\hline Q6 & $\begin{array}{l}\text { hasLeafDivision, hasLeafPart, } \\
\text { hasLeafHabit, hasLeafApices }\end{array}$ & 14 & 21 & 0.6667 & hasLeafApices & 14 & 14 & 1.0000 \\
\hline Q7 & $\begin{array}{c}\text { hasTraditionalUses } \\
\text { hasHerb } \\
\text { hasRootHabit }\end{array}$ & 1 & 5 & 0.2000 & hasRootHabit & 1 & 1 & 1.0000 \\
\hline Q8 & $\begin{array}{c}\text { hasStemType } \\
\text { hasGeneralHabit } \\
\text { hasStemHabit }\end{array}$ & 14 & 18 & 0.7778 & hasStemType & 14 & 14 & 1.0000 \\
\hline Q9 & $\begin{array}{c}\text { hasEnglishVernacularName } \\
\text { hasTasteCharater }\end{array}$ & 21 & 30 & 0.7000 & hasTasteCharacter & 21 & 29 & 0.7241 \\
\hline Q10 & $\begin{array}{c}\text { hasEnglishVernacularName } \\
\text { hasGeneralHabit }\end{array}$ & 21 & 22 & 0.9545 & hasGeneralHabit & 21 & 21 & 1.0000 \\
\hline Mean & & & & 0.6314 & & & & 0.9088 \\
\hline
\end{tabular}

* $\mathrm{MMO}=$ Multi-modality $\quad$ ontology, $\mathrm{C}=$ Correct, $\mathrm{R}=$ Retrieved, $\mathrm{P}=$ Precision, $\mathrm{OP}=$ Object Properties, $\mathrm{OPF}=\mathrm{Object}$ Properties Filter

The accuracy of the proposed IMR was assesed by using precision measurement. Table 2, summarizes the results achieved. The results between the semantic multi-modality ontology IMR using object properties filter (MMO-IMROPF) and the semantic multi-modality ontology IMR without using object properties filter (MMO-IMR) were compared. Each of the precision numbers from Q1 to Q10 showed encouraging enhancement. The mean results showed almost $30 \%$ improvement of precision by utilizing the object properties filter.

Based on Table 6.3, the results of the 10 samples of queries in MMO-IMR were classified into three categories. The three categories were low precision, moderate precision and high precision. Q5 and Q7 were categorized in the low precision measurement where the values of precision were $33 \%$ and $20 \%$ respectively. The eight images in Q5 that were related to hasDefinition, hasShapeLeaf and hasShapeFlower object properties were not relevant to this query. The precision of this query was relatively low below $50 \%$ due to the majority of the non-relevant images retrieved in the results. Similarly, the majority or $80 \%$ of the images retrieved in Q7 were not relevant. The precision measurement was also below $50 \%$ which was relatively low. 
Seven queries could be categorized as moderate precision. Moderate precision was classified between more than or equal to $50 \%$ and less than $80 \%$ precision measurement. The queries were Q1, Q2, Q3, Q4, Q6, Q8 and Q9. In Q1, seven images related to hasLeafPart, hasLeafHabit and hasLeafDivision were not relevant to this query. In Q2, three images related to hasFruitHabit, hasLeafHabit and hasSynonym were not relevant for this query. Even though hasOdourCharacter was chosen in this query, the precision measurement still could not achieve $100 \%$. It is due to the distraction of the word 'smell' in the query sentence where there were still 10 non-relevant images in the final result. As opposed to Q3, the utilization of the object properties filter could not provide better results as only one object property was related to this query. The result remained unchanged as before using the object properties filter approach. The precision measurement could not achieve $100 \%$ because there were still three non-relevant images in the final result. In Q4, Q6 and Q8 nine images related to hasLeafHabit, hasLeafPart, hasShapeFlower, hasHerb and hasLeafDivision object properties, seven images related to hasLeafDivision, hasLeafHabit and hasLeafPart object properties, and four images related to hasGeneralHabit and hasStemHabit were not relevant to this query, respectively. Finally, in Q9, only one image related to hasEnglishVernacularName was not relevant to this query. Although the selection of hasTasteCharacter was chosen in this query, the precision measurement still could not achieve $100 \%$. It was due to the distraction of the word 'taste' in the query sentence where, there were still eight non-relevant images in the final result.

Among the 10 queries in MMO-IMR, only Q10 could be categorized as high performance precision. The high performance precision was classified as between more than or equal to $80 \%$ and less than or equal to $100 \%$. In Q10, the precision value was $95 \%$. In this query, only one image related to hasEnglishVernacularName was not relevant to this query.

The main reason why the mean value of MMO-IMR was $63 \%$ was because the metadata contained similar names and was located in different classes which would reduce the performance of precision results. Therefore, the object properties filter approach could be utilized to screen more accurate retrieval results and improve the performance of the precision. In MMO-IMR-OPF, the majority or $70 \%$ of the queries achieved high precision value which was $100 \%$. The queries were Q1, Q4, Q5, Q6, Q7, Q8 and Q10. They contributed as a main factor of the high precision mean results based on the selection object properties of hasLeafMargins, hasShapeLeaf, hasShapeFruit, hasLeafApices, hasRootHabit, hasStemType and has GeneralHabit respectively. However, Q2 and Q9 did not achieve 100\% precision but still indicated an improvement in precision measurement by utilizing the proposed approach. The precision of 
Q2 and Q9 increased 6\% and 2\% respectively. Finally, in Q3, the utilization of the object properties filter approach was not effective due to the one object property for this query only. The precision result was unchanged; it was the same as before using the object properties filter approach.

\section{CONCLUSION}

This paper presented the enhanced multi-modality ontology IMR by incorporating the object properties filter. The empirical results of the proposed multi-modality ontology IMR produced more accurate results in terms of precision. Object properties filter played an important role in increasing the accuracy of IMR, effectively. Through the experiment, it was observed that, without using the object properties filter, the users were still able to retrieve the images as they wanted but the number of potential retrieved images varied especially when the results of the queried images were enormous. Therefore, the multi-modality ontology IMR incorporated the object properties filter to maximize the possibility of obtaining precise targeted images.

\section{REFERENCES}

Broilo, M., \& Natale, F. G. B. De. (2010). A stochastic approach to image retrieval using relevance feedback and particle swarm optimization. IEEE Transactions on Multimedia, 12(4), 267-277.

Chatzichristofis, S. A., Zagoris, K., Boutalis, Y. S., \& Papamarkos, N. (2010). Accurate image retrieval based on compact composite descriptors and relevance feedback information. International Journal of Pattern Recognition and Artificial Intelligence, 24(02), 207-244. http://doi. org/10.1142/S0218001410007890

Commitee, M. H. M. (2009). Malaysian herbal monograph (Volume II). Kuala Lumpur, Malaysia: Forest Research Institute Malaysia (FRIM).

Commitee, M. H. M. (2012). Malaysian herbal monograph (Volume III). Shah Alam, Malaysia: Malaysian Herbal Monograph Commitee.

Cox, I. J., Miller, M. L., Minka, T. P., Papathomas, T. V., \& Yianilos, P. N. (2000). The Bayesian image retrieval system. PicHunter: Theory, implementation and psychophysical experiments. IEEE Transactions on Image Processing, 9(1), 20-37. http://doi.org/10.1109/83.817596 
Gruber, T. R. (1993). A translation approach to portable ontology specifications. Journal of Knowledge Acquisition, 5(2), 199-220.

Harris, J. G., \& Harris, M. W. (2004). Plant identification terminology: An illustrated glossary $\left(2^{\text {nd }}\right.$ ed.). Spring Lake Publishing.

Hoi, S. C. H., Lyu, M. R., \& Jin, R. (2006). A unified log-based relevance feedback scheme for image retrieval. IEEE Transactions on Knowledge and Data Engineering, 18(4), 509-523. http://doi.org/10.1109/ TKDE.2006.1599389

Ismail, Z., Ismail, N., \& Lassa, J. (1999). Malaysian herbal monograph (Volume I). Kuala Lumpur, Malaysia: Malaysian Monograph Committe.

Kesorn, K. (2010). Multi-modal mult- semantic image retrieval. (Doctoral Thesis, University of London). Retrieved from https:/qmro.qmul. ac.uk/xmlui/handle/123456789/438

Khalid., Y. I. A. M., Azman, S., \& Noah, M. (2012). Improving the performance of multi-modality ontology image retrieval system using DBpedia. Procedia Information Technology \& Computer Science p. 1-9.

Khodaskar, A., \& Ladhake, S. (2015). New-fangled alignment of ontologies for content-based semantic image retrieval. In Procedia Computer Science (Vol. 48, pp. 298-303). Elsevier Masson SAS. http://doi.org/10.1016/j. procs.2015.04.185

Kim, D., \& Chung, C.-W. (2003). Qcluster: Relevance feedback using adaptive clustering for content-based image retrieval. In Proceedings of the ACM SIGMOD International Conference on Management of Data (pp. 599-610).

Kovashka, A., Parikh, D., \& Grauman, K. (2015). WhittleSearch: Interactive image search with relative attribute feedback. International Journal of Computer Vision, 115(2), 185-210. http://doi.org/10.1007/s11263-0150814-0

Kurita, T., \& Kato, T. (1993). Learning of personal visual impression for image database systems. Proceedings of 2nd International Conference on Document Analysis and Recognition (ICDAR '93). http://doi. org/10.1109/ICDAR.1993.395676 
Liu, Y., Zhang, D., Lu, G., \& Ma, W. Y. (2007). A survey of content-based image retrieval with high-level semantics. Pattern Recognition, 40(1), 262 - 282. http://doi.org/10.1016/j.patcog.2006.04.045

M. Riad, A., K. Elminir, H., \& Abd-Elghany, S. (2012). A literature review of image retrieval based on semantic concept. International Journal of Computer Applications, 40(11), 12-19. http://doi.org/10.5120/5008-7327

Magesh, N., \& Thangaraj, P. (2011). Semantic image retrieval based on ontology and SPARQL query. International Journal of Computer Applications, 1(3), 12-16.

Mezaris, V., Kompatsiaris, I., \& Strintzis, M. G. (2003). An ontology approach to object-based image retrieval. International Conference on Image Processing, ICIP (pp. 511-514).

Noy, N. F., \& McGuinness, D. L. (2000). Ontology development 101 : A guide to creating your first ontology. Retrieved from http://protege.stanford. edu/publications/ontology_development/ontology101.pdf

Rocchio, J. J. (1965). Relevance feedback in information retrieval, 293-305. http://doi.org/10.1145/1463891.1463926

Rocchio, J. J. (1966). Document retrieval systems - optimization and evaluation. Harvard University.

Rui, Y., Huang, T. S., \& Chang, S.-F. (1999). Image retrieval: Current techniques, promising directions, and open issues. Journal of Visual Communication and Image Representation, 10, 39-62.

Rui, Y., Huang, T. S., \& Mehrotra, S. (1997). Content-based image retrieval with relevance feedback. Proceedings of Image Processing pp.815-818

Saian, R., \& Ku-Mahamud, K. R. (2004). Searching malay text using stemming algorithm. Journal of Information and Communcation Technology (JICT), 3(2), 107-117. http://doi.org/10.1017/CBO9781107415324.004

Serrano, N., Savakis, A. E., \& Luo, J. (2004). Improved scene classification using efficient low-level features and semantic cues. Pattern Recognition, 37(9), 1773-1784. http://doi.org/10.1016/j.patcog.2004.03.003 
Singh, P., Goudar, R., Rathore, R., Srivastav, A., \& Rao, S. (2013). Domain ontology-based efficient image retrieval. Proceedings of 7 th International Conference on Intelligent Systems and Control.

Sivakamasundari, G., \& Seenivasagam, V. (2012). Different relevance feedback techniques in cbir: a survey and comparative study. International Conference on Computing, Electronics and Electrical Technologies (pp. 1115-1121).

Smeulders, A. W. M., Worring, M., Santini, S., Gupta, A., \& Jain, R. (2000). Content-based image retrieval at the end of the early years. IEEE Transactions On Pattern Analysis And Machine Intelligence, 22(12).

Su, J., Huang, W., \& Yu, P. S. (2011). Efficient relevance feedback for content-based image retrieval by mining user navigation patterns. IEEE Transactions on Knowledge and Data Engineering, 23(3), 360-372.

Uschold, M., \& Gruninger, M. (1996). Ontologies : principlesdd, methods and applications. Knowledge Engineering Review, 2, 1-53.

Wang, H., Liu, S., \& Chia, L.-T. (2008). Image retrieval with a multi-modality ontology. Journal of Multimedia Systems, 13, 379-390. http://doi. org/10.1007/s00530-007-0099-4

World Health Organisation (WHO). (2008). Traditional medicine. Retrieved from http://www.who.int/mediacentre/factsheets/fs134/en/\#

Zhang, D., Islam, M. M., \& Lu, G. (2012). A review on automatic image annotation techniques. Journal of Pattern Recognition, 45(1), 346-362. http://doi.org/10.1016/j.patcog.2011.05.013

Zhang, Y. J. (2007). Semantic based visual information retrieval. IRM Press.

Zhou, X. S., \& Huang, T. S. (2003). Relevance feedback in image retrieval: A comprehensive review. Multimedia Systems , 8, 536-544. http://doi. org/10.1007/s00530-002-0070-3 